\title{
A Petri net approach to assess the effects of railway maintenance on track availability
}

Author 1

- Claudia Fecarotti, Assistant Professor

- Department of Industrial Engineering \& Innovation Sciences, Eindhoven University of Technology, Eindhoven, Netherlands

- ORCID number

Author 2

- John Andrews, Professor

- Department of Mechanical Engineering, Faculty of Engineering, The University of Nottingham, Nottingham, UK

- ORCID number

Corresponding author: Claudia Fecarotti, e-mail: c.fecarotti@tue.nl

\section{Abstract}

The railway infrastructure includes a portfolio of assets which are subjected to degradation and failure processes due to both usage and aging. As a consequence of degradation and failures, speed restrictions and line closures may be imposed to control the risk of derailment. Such actions have a direct impact on service as they lead to delays and journey cancellations. Maintenance is implemented to control the state of the assets. Different maintenance strategies determine different asset conditions and performance profiles, and consequently a different impact on service. This paper presents a simulation tool based on Petri nets, which combines degradation and maintenance processes to predict the future track geometry conditions, including the probability of those failure modes leading to speed restrictions and line closures. Such a model is a valuable feature of an effective infrastructure asset management system which intends to support cost-effective informed decisions on railway maintenance.

\section{Keywords chosen from ICE Publishing list}

Railway Systems; Maintenance \& Inspection; Mathematical Modelling.

\section{List of notation}


$T_{i} \quad$ transition node in the Petri net

$P_{i} \quad$ place node in the Petri net

$\sigma_{o p} \quad$ threshold of standard deviation for track vertical alignment inducing opportunistic maintenance

$\sigma_{r m} \quad$ threshold of standard deviation for track vertical alignment inducing routine maintenance

$\sigma_{s r} \quad$ threshold of standard deviation for track vertical alignment inducing speed restriction

$\sigma_{l c} \quad$ threshold of standard deviation for track vertical alignment inducing line closure

$\sigma_{g, o p} \quad$ threshold of standard deviation for track gauge inducing opportunistic maintenance

$\sigma_{g, r m} \quad$ threshold of standard deviation for track gauge inducing routine maintenance

$\sigma_{g, e r} \quad$ threshold of standard deviation for track gauge inducing emergency maintenance

$\sigma_{g, \text { ir }} \quad$ threshold of standard deviation for track gauge inducing immediate maintenance

$\beta, \eta \quad$ parameters of Weibull distribution, shape parameter and scale parameter respectively 
2 The ultimate aim of a railway system is the transport of people and goods at the

\section{Introduction}

required level of service and safety. The railway comprises a variety of heterogeneous assets which are subject to degradation and failures due to usage and aging. As a consequence of degradation and failures, speed restrictions and line closures may be imposed to control the risk of derailment. Such actions have a direct impact on service as they lead to delays and journey cancellations. To control the state of the assets maintenance strategies must be developed which specify the inspection and intervention activities to be performed, and the rules for their implementation. Different strategies will determine different asset conditions and performance profiles, and consequently a different impact on service. The capability to evaluate such an impact is paramount for a cost-effective planning of maintenance interventions. In (Burkhalter, Martani, \& Adey, 2018) the impact of speed restrictions or line closures being imposed is considered when computing the costs and benefits of intervention plans. Here the probabilities of an object requiring a speed restriction or a line closure is computed by means of fault tree analysis (an object being either a component, such as a bridge or a switch, or a track section) and used to evaluate the risk related to a given intervention program. In (Moreu, Spencer Jr., Foutch, \& Scola, 2017) the authors develop a framework to prioritise maintenance interventions on railroad bridge networks. The operational costs associated to the probability that the bridges will exceed the "service limit states" depending on the maintenance decision are minimised.

This paper presents a modelling approach based on the Petri net method, which combines the degradation, failure and intervention processes to predict the future track geometry conditions, including the probability of those failure modes leading to speed restrictions and line closures. The approach enables the asset response to a variety of potential maintenance strategies to be investigated and predicted. The model is statebased, where states which are relevant from a maintenance perspective are explicitly 
modelled. Of those states, the ones which correspond to the imposition of speed

restrictions and line closures are particularly relevant for their impact on service. Due to the stochastic nature of the modelled processes, stochastic simulation via the Monte Carlo method is the most appropriate approach to analyse and solve the model.

Statistics are collected on the probability that the asset is in any of the modelled states and on the number of interventions performed during a given time horizon. With such models, a wide range of maintenance strategies can be analysed. The resulting probability, number and duration of speed restrictions and line closures are an indicative measure of the effects that a given maintenance strategy will have on service. Such statistics can be then used directly, or within an optimisation procedure, to support the planning and development of maintenance strategies to achieve given service performance targets.

\subsection{Track geometry degradation and maintenance}

Track geometry, both vertical and horizontal, strongly affects the quality and safety of the ride. Track geometry is periodically inspected by running Track Recording Vehicles along the network. The vehicle measures the location of the rails and provides the variations of the rails vertical and horizontal position, gauge, twist and cyclic top over 1/8th mile section. Measurements, particularly the ones related to the vertical alignment, are then used to categorise the track in terms of quality bands, to plan maintenance and, if necessary, to take actions such as speed restrictions and line closure to control the risk of derailment. The track's components responsible for keeping the required track geometry are the ballast, sleepers and fastenings. Specifically, while vertical alignment defects are mainly attributable to degradation of

51 the ballast, gauge, horizontal alignment, cross-level and twist defects are mainly due to 52 degradation and failure of sleepers and fastenings. Gauge widening is the effect of multiple sleepers/fastenings failures. To maintain those components means to keep track geometry to acceptable levels. Although the degradation and maintenance 
processes affecting these components are very different, many dependencies arise due to common processes such as inspection, opportunistic maintenance and combined renewal. However, most of the models presented in the literature address the modelling of degradation and maintenance of each component individually. In order to represent different degradation states, the transitions between these states and the restorative actions provided by the maintenance processes, the model must accommodate a state-based approach. Due to the variability of the times for degradation and maintenance to occur it should also be stochastic. The main approaches utilised in the literature to model degradation and maintenance are Monte Carlo simulation methods using either statistical models or state-based models to describe the asset degradation and the effects of maintenance activities. As an example of state-based approaches, Markov models have been developed in Meier Hirmer et al. (2009), Bai et al. (2013) and Prescott and Andrews (2013a) to represent track geometry degradation and its maintenance processes. Markov-based models are stochastic models capable of describing dynamic systems for which future states depend only on the current state. The history of what has occurred in the past is irrelevant to future behaviour. The size of a Markov model can increase considerably with the number of components to consider. An alternative modelling technique to the Markov approach is the Petri net (PN) method. PNs are a formalisms to model discrete event dynamic systems with concurrencies and dependencies (Murata, 1989; David and Alla, 2010). Andrews (2012) presents a PN to model ballast degradation and maintenance for a $1 / 8^{\text {th }}$ of mile section of track. The author first analyses track geometry data from inspection and maintenance records so to evaluate the distribution of times to degrade from/to different states of interest from a maintenance perspective.

79 These distributions are then used to sample the times to degrade of the ballast. A 80 similar model is presented by Prescott and Andrews, (2013b) who develop a PN combining degradation, inspection, maintenance and renewal processes for ballast on 
a railway network with different regions each one supervised by a regional maintenance engineer. Andrews et al. (2014) apply the previously developed PN model to predict the state of the track geometry over any specified asset management strategy. In addition the model is capable of computing the expected whole life costs. In (Lake et al., 2000a,b) the authors develop a simulation model to predict the distribution of groups of failed timber sleepers in a track section for different renewal strategies. The sleepers' lifetime is assumed to follow a 2-parameter Weibull distribution, and the renewal strategies are based on the minimum number of adjacent sleepers failed and the frequency of intervention. The same model is applied in (Yun and Ferreira, 2003) to a track with 1000 sleepers. Zhao et al. (2007) evaluate the reliability of a segment of sleepers modelled as a k-out-of-n system, where the lifetime of the sleepers is assimilated to a 2-parameter Weibull distribution.

The aforementioned models focus on individual components of the track system, either the ballast or the sleepers. Therefore, dependencies induced by common processes such as inspection, opportunistic maintenance and combined renewal of multiple components and adjacent track sections would require a framework that combines the individual models into one. A modular representation is presented in this paper, where independent modules for modelling the degradation of the vertical alignment and gauge are then combined to predict track geometry response to maintenance. The models include a representation of the degraded states and corresponding actions that have a direct impact on service. These are conditions that, according to the stakeholders' policies, require the imposition of speed restrictions or line closures.

\section{Track geometry model}

105 The track geometry model consists of two modules which represent vertical geometry 106 and gauge degradation respectively, and the corresponding maintenance actions that 107 can be performed. Variations in vertical alignment is mainly due to ballast degradation, while sleepers and fastenings failures are mainly responsible for gauge spreading. The 
modelling approach adopted is based on the PN method. In the following, a brief

110 introduction on PN is given to enable a better understanding of the proposed model.

\section{$111 \quad 2.1$ The Petri net method.}

112 A PN (Murata, 1989; David and Alla 2010) is a bi-partite graph with nodes called places

113 and transitions. Places are represented as circles while transitions are represented as

114 rectangles. Places model possible states for a component/system, while transitions

115 model events that cause the system state to change. Input and output arcs connect

116 places to transitions and vice versa, and are represented by arrows. Tokens are held in

117 places; the number of tokens in a place $P_{i}$ is called marking of $P_{i}$ and is indicated by

$118 m_{P i}$. The number and distribution of tokens across the PN, called marking of the PN,

119 represent the system state at a given time. Transitions are responsible for "consuming"

120 tokens from the input places, and "producing" tokens into the output places thus

121 determining a change in the marking. This is referred to as "firing" of the transition and

122 corresponds to the occurrence of the event modelled by the transition. The number of

123 tokens consumed and produced depends on the multiplicity of the input and output arcs respectively. An additional type of arc called inhibitor is often used to forbid the firing of a transitions under given conditions. Inhibitor arcs are as arcs with a circle end rather than an arrow end. The rules according to which transitions fire are as follows:

- First, a transition is enabled to fire if (1) the number of tokens in each input

- When firing, the transition "consumes" a number of tokens from the input places equal to the input arcs multiplicity, and "produce" a number of tokens into the output places equal to the output arcs multiplicity.

134 Figure 1 gives an example of a simple PN where transition T1 has two input places P1 135 and P3, one place connected by inhibitor arc, P2, and two output places P4 and P5. 
136 Examples of marking which do not enable T1 are given in Figure 1-a and 1-b, while

137 firing is enabled for marking in Figure 1-c.

138 FIGURE 1 HERE

139

140

141

142

143

144

145

146

147

148

149

150

151

152

153

154

155

156

157

158

159

160

161

162

163

164

Figure 1 PN with different possible markings.

Transitions can be immediate or timed. Timed transitions, once enabled, will only fire when a given firing time interval has elapsed. Figure 2 shows a PN with timed transition T1 and associated firing time interval $\Delta t$ before firing (2-a) at a given time $t$, and after firing (2-b) at time $t+\Delta t$.

FIGURE 2 HERE.

Figure 2 PN with timed transition, before (a) and after (b) firing.

The firing time interval can be either deterministic or stochastic. Events or processes affected by randomness, such as the degradation or failure of a component, are modelled by stochastic transitions whose firing time intervals are sampled from appropriate stochastic distributions. Multiple distributions can be associated to the same transition, and the appropriate one is selected based on the marking of specific places called conditional places. In a PN representation, a transition is linked to its conditional place by a dotted arrow. This feature is useful to represent events whose distribution of times of occurrence changes depending on some given condition. The mode in which a transition fires, referred to as firing mode, can be standard or nonstandard. According to a standard firing mode, a transition consumes and produces tokens only according to the multiplicity of the input and output arcs. If a non-standard firing mode is associated to a transition, then the new marking is evaluated according to a given firing mode function. An explanatory example of a PN where a transition has a non-standard firing mode is given in Figure 3. The PN consists of one transition T1, with one input place, P1, three output places, P2 to P4, and one conditional place, P5. FIGURE 3 HERE 
$\mathrm{T} 1$ is a timed stochastic transition whose firing time $t$ can be sampled from one out of two Weibull distributions $W_{1}\left(\beta_{1}, \eta_{1}\right)$ and $W_{1}\left(\beta_{1}, \eta_{1}\right)$ depending on the marking of conditional place $\mathrm{P} 5, m_{P 5}$. The new marking resulting from firing of $\mathrm{T} 1$ is also random.

171 Each output place has a given probability $\alpha_{i}$ of receiving a token after firing of T1. When

172 T1 fires, it will sample a random number $p$ from a uniform distribution between 0 and 1.

173 Depending on the value $p$, a token will be added to one out of the three output places

$174 \mathrm{P} 2$ to $\mathrm{P} 4$. Such type of transition is called routing probabilistic transition. It can be used to represent maintenance actions whose effectiveness is uncertain. The symbols used to represent the different types of transitions are showed in Figure 4.

177 FIGURE 4 HERE.

Figure 4 Symbols used for different types of transitions.

2.2 Model for vertical alignment: ballast

181 The degradation of the ballast is modelled as a phased process. The conditions of the

182 ballast are implied by the values of the standard deviation (SD) of the vertical alignment 183 provided by the Track Recording Vehicles. The SD of the vertical alignment is therefore considered the indicator of the ballast conditions. Once degraded conditions are revealed by inspection, then the appropriate maintenance action is scheduled and performed at the required time. The model therefore includes ballast deterioration,

187 inspection, routine and opportunistic maintenance, and emergency repair.

188 Degradation process. Figure 5 represents the degradation process.

189 FIGURE 5 HERE

190 Figure 5 PN representing ballast degradation.

192 Degradation is modelled as a phased process where a number of discrete states which 193 are relevant from a maintenance perspective are considered. These states are 
194 represented by places P1 to P7. Each state represents a severity degradation level and 195 is characterised by a threshold value for the SD of the vertical alignment. If the 196 corresponding SD value is reached, then the state is entered. A different urgency of 197 intervention is associate to each degradation level. Three approaches to maintenance 198 are modelled, namely routine, opportunistic and emergency (or corrective) maintenance. Routine maintenance is a scheduled intervention often planned weeks or 200 months ahead of execution. Opportunistic maintenance means that once a routine 201 intervention is going to be carried out on a given section, adjacent sections which are in 202 a condition close enough to require routine maintenance will be also included in the 203 intervention. Finally, an emergency intervention is carried out when inspection reveals 204 a degraded condition which could potentially cause a derailment. In such circumstances a speed restriction or even a line closure is imposed immediately, and 206 intervention is performed as soon as possible. Place P1 indicates new conditions (or 207 following renewal). Place P2 represents a state where opportunistic maintenance is 208 possible. The corresponding threshold of the SD is $\sigma_{\mathrm{op}}$. Place P3 models a state 209 requiring routine maintenance to be scheduled and performed within a given period of time. The SD threshold is $\sigma_{\mathrm{rm}}$. Place P5 and P6 represent two levels of degradation

211 such that a speed restriction or line closure respectively must be imposed to control the 212 risk of derailment while an emergency intervention is scheduled. These are very 213 undesirable states that, if revealed, cause a disruption to the railway service, whereas if 214 not detected could constitute potentially hazardous situations. P5 and P6 are entered 215 when the threshold values $\sigma_{\mathrm{sr}}$ or $\sigma_{\mathrm{lc}}$ are exceeded respectively. It is possible that, if the 216 inspection process reveals that the track is in a state which will soon reach $\sigma_{\mathrm{sr}}$, an 217 emergency repair might be carried out to avoid reaching the undesirable state requiring 218 speed restriction. This state is represented by place P4 with threshold $\sigma_{\text {crit. }}$ After 219 maintenance, track geometry is never restored to as good as new conditions. Place P7 220 is used here to indicate the best possible state achievable following repair. The time to 
degrade from one state to the next depends on the value chosen for each SD threshold and is ruled by stochastic transitions T1 to T6. A set of firing time distributions is associated to each of these transitions. The distribution of times to degrade from one state to the next depends on the value of these thresholds. Therefore, depending on the SD threshold, the appropriate distribution is selected for each of transitions $\mathrm{T} 1$ to T6. The SD thresholds triggering a speed restriction or a line closure for a given track category are usually fixed for safety reasons. The thresholds for opportunistic $\sigma_{o p}$ and routine maintenance $\sigma_{\mathrm{rm}}$ instead can be varied to investigate the effects of more or less conservative approaches to condition-based maintenance on the track long-term behaviour. Different values of $\sigma_{\text {op }}$ determine different distributions of times to degrade associated to transitions $\mathrm{T} 1, \mathrm{~T} 2$ and $\mathrm{T} 6$, while different values of $\sigma_{\mathrm{rm}}$ determine different distributions associated to transitions T2 and T3 (or T4 if the critical state coincides with the state requiring a speed restriction). In order to automate the selection of different values for $\sigma_{\mathrm{op}}$ and $\sigma_{\mathrm{rm}}$, and the appropriate distributions for the corresponding transitions, places P15 and P16 are introduced (Figure 6). If a correspondence is established between their marking and given values of $\sigma_{\mathrm{op}}$ and $\sigma_{\mathrm{rm}}$, then $\mathrm{P} 15$ and $\mathrm{P} 16$ can be used as conditional places for transitions T1 to T4, and T6. For example, if two potential values are considered for $\sigma_{\mathrm{op}}$, then two distributions are associated to each of transitions T1, T2 and T6. Depending on the marking of P15, the appropriate distribution will be selected between the available two for each of the above transitions. FIGURE 6 HERE

Figure 6 PN accounting for different SD thresholds triggering opportunistic and routine maintenance.

Inspection process. The periodic inspection process is represented by loop P19-T18P20-T17-P19 in Figure 7.

Figure 7 PN describing ballast degradation and inspection. 
250 When inspection is not performed, place P20 is marked while place P19 is empty; such

251 marking will enable transition T17 that will fire after the specified time interval $\vartheta 1$.

252 Transition T17 is a timed deterministic transition and the time interval $\vartheta 1$ depends on

253 the marking of place P18 which defines the inspection strategy. By firing, T17 will

254 remove the token from place P20 and add a token in place P19 indicating that the track

255 is now under inspection and degraded states, if any, can be revealed. Places P8 to

256 P12 represent the revealed states corresponding to each possible degraded condition.

257 Intervention processes. Only once a degraded condition has been revealed,

258 maintenance can be scheduled and carried out with different urgency depending on the

259 level of degradation detected. The PN in Figure 8 includes the intervention activities

260 that can be performed on the ballast to restore geometry conditions.

261 FIGURE 8 HERE

262 Figure 8 PN describing ballast degradation, inspection and maintenance processes.

263

264 These are represented by transitions T12 to T16. Specifically, T12 to T14 indicate the

265 imposition of a speed restriction or line closure, and the scheduling and execution of an

266 emergency intervention. T15 and T16 represent the scheduling and execution of a

routine intervention. In order to account for the randomness in the effectiveness of

268 tamping, the output state after firing of T16 (execution of routine tamping) is randomly

269 selected among places P2, P3, P4 and P7. Transition T16 will therefore add a token to

270 one of places P2, P3, P4 and P7, each with a given probability. Since the effectiveness

271 of tamping strongly depends on the maintenance history, this probability changes with

272 the number of tamping intervention performed, and thus depends on the marking of

273 place P14. The latter is simply used to count the number of tamping that have been

274 performed. In case of an emergency intervention which is often a manual tamping, a

275 good state (place P7) is usually restored. It is worth specifying that the model explicitly

276 represents speed restrictions and line closures due to unplanned maintenance, namely

277 emergency interventions that are triggered when track geometry has degraded above a 
278 given limit. At its current stage the model does not explicitly account for the section

279 closures to carry out routine maintenance, nor the speed restrictions that are often

280 imposed after a renewal as these are considered as 'planned'.

281 Since the ballast degradation rates increases with the number of tamping interventions

282 previously performed, the marking of place P14 will affect the parameters of the

283 distributions associated to transitions T1 to T6. The time for ballast renewal depends

284 on the renewal strategy adopted. Renewal can be based on age or maintenance

285 history. In the first case, the ballast is renewed after a fixed number of years, and

286 transition T35 is used, with a deterministic firing time equal to the ballast lifetime. If

287 renewal is based on the past maintenance, then the ballast is renewed as soon as a

288 maximum number of tamping interventions are performed. Transition T37 is used,

289 which is enabled as soon as the marking of place P14 reaches the threshold $\mathrm{N}_{\text {tamp,max. }}$

290 A third renewal strategy can be considered, according to which ballast is renewed as

291 soon as the sleepers in the same sections are recommended for renewal. Transition

292 T36 is used in this case; it provides a link between the ballast PN module and the PN

293 module for sleepers presented in the next section. Place P37 is a conditional place for

294 the renewal transitions T35, T36 and T37. Depending on its marking, one of the three

295 renewal options can be selected. For example, if marking of P37 is 1, then transition

296 T35 is enabled, namely ballast renewal is based on ballast age. If marking of P37 is 2,

297 then transition T37 is enabled, namely ballast renewal is based on past maintenance.

298 Finally, if marking of P37 is 3, then transition T36 is enabled, namely the ballast is

299 renewed when all sleepers (and consequently fastenings as well) within the $1 / 8^{\text {th }}$ mile

300 section are scheduled for renewal. The conditions for sleepers/fastenings renewal are

301 described in the following section.

$302 \quad 2.3$ Model for track gauge: sleepers and fastenings.

303 This model represents the effects of sleepers and fastenings failures on gauge

304 widening. Individual ineffective sleepers or elements of the fastening system do not 
have a direct effect on gauge widening. It is only when a number of elements in a given

306 length of track are ineffective that the gauge will actually spread to a level that will eventually lead to a potential derailment risk. Inspection by Track Recording Vehicles only reveals group of failed components that have already caused the gauge to spread, while detection of isolated failed elements relies on visual inspection. Possible interventions are replacement of clips and/or rail pads, and spot re-sleepering.

311 Degradation process. The different degraded states in the PN, correspond to different 312 levels of gauge widening requiring intervention with different levels of urgency. Each level corresponds to a given number of ineffective elements within a certain length of track (Figure 9). Failure dependencies between sleepers and fastenings are not currently accounted for in the model.

FIGURE 9 HERE

\section{Figure 9 PN for gauge degradation.}

The considered section ( $1 / 8^{\text {th }}$ of mile) is therefore divided into clusters of consecutive sleepers/fastenings. If the assumption is that a failed sleeper has the same effect on gauge widening as a pair of failed fastenings, then the cluster size is equivalent to the number of consecutive sleepers and/or fastenings whose failure will determine a line closure. Here, the clusters' size corresponds to a single track length containing 10 consecutive sleepers and 20 fastenings ( 2 per sleeper). As $1 / 8^{\text {th }}$ of a mile single track section contains typically 300 sleepers (and corresponding 600 fastenings), it follows that each $1 / 8^{\text {th }}$ of a mile section contains 30 clusters. The model currently assumes that gauge widening is due to a failure of multiple elements within the same cluster, but does not account for the situation when elements at the edges of two adjacent clusters fail. Coloured tokens are used to represent each cluster which is defined by the following attributes: an ID to uniquely identify the cluster, the cluster's size (as defined previously), the number of working sleepers $\mathrm{N}_{\mathrm{sl}, \mathrm{s}}$ and fastening components $\mathrm{N}_{\mathrm{t}, \mathrm{s}}$, the number of ineffective sleepers $\mathrm{N}_{\mathrm{sl}, \mathrm{i}}$ and fastening components $\mathrm{N}_{\mathrm{f}, \mathrm{i}}$, the total number of 
333 ineffective elements $N_{\text {tot, }, \text {. }}$ The IDs are given to each cluster in order, e.g. $1,2,3 \ldots$ so

334 that it is possible to identify adjacent clusters. The number of working components in 335 each cluster can decrease over time, while sleepers and/or elements of the fastenings

336 become ineffective. The value of each token is therefore updated every time a

337 component fails, by decreasing the number of working elements and increasing the 338 number of failed elements. In order to avoid confusion with the standard tokens, the 339 coloured tokens defined above are referred to as token-cluster. Five levels of gauge 340 widening have been considered and are represented by places P21 to P25. Thresholds

$341 g_{\mathrm{op}}, g_{\mathrm{rm}}, g_{\mathrm{er}}$ and $g_{\mathrm{ir}}$ are associated to places to P22 to P25 and correspond to gauge 342 conditions requiring opportunistic, routine, emergency and immediate interventions.

343 P21 corresponds to no gauge widening. Degradation from one state to the next is caused by failure of components in the same cluster. When a specified number of

345 components within the same cluster have failed, then the corresponding token-cluster

346 is moved to the next degraded state through the transition.

347 Inspection process. The inspection process, depicted in Figure 10, has the same 348 features as described above for the ballast module, except that here also visual 349 inspection can be considered (loop P32-T31-P33-T32). FIGURE 10 HERE

Figure $10 \mathrm{PN}$ for gauge degradation and inspection.

353 Upon inspection the current gauge level is revealed. This is represented by one or 354 more among transitions T23 to T26 firing and adding a token-cluster to the 355 corresponding output place (P26 to P29). Intervention process. Once the gauge level has been revealed, then maintenance is scheduled and performed, this being modelled by means of transitions T27 to T30 as

358 shown in Figure 11.

359 FIGURE 11

360 Figure $11 \mathrm{PN}$ for gauge degradation, inspection and maintenance. 
362 Transitions T28 to T30 represent routine, emergency and immediate interventions 363 respectively. T27 models the opportunistic maintenance which is only possible if a 364 routine intervention is already planned to replace an adjacent group of sleepers/fastenings. Places P30 and P31 are used to simply keep track of the number of sleepers and fastenings replaced respectively. When an intervention is scheduled, only failed components are replaced. This means that functioning fastenings holding a failed sleepers are not replaced along with the sleeper; this however is not always the case in reality. Two renewal policies are considered, either based on age or conditions. Transition T33 represent age-based renewal and it fires when the sleepers lifetime is reached. T34 models a condition-based renewal. This transitions 'checks' the marking of places P26 to P29. Specifically, places P26 to P29 will (potentially) contain one or more token-cluster. For each of these places, the number of failed sleepers contained in each cluster is counted. If the overall number of ineffective sleepers is above a given threshold, then renewal is recommended. Place P35 is a conditional place for both transitions T33 and T34, and its marking determines the renewal strategy to be selected. For example, marking of P35 equal to 1 corresponds to time based renewal, thus transition T33 is enabled. If marking of P35 is 2 , then the selected renewal 379 strategy is based on conditions, thus transition T35 is enabled. After renewal (firing of 380 either T33 or T34) the overall state of the system is reset to new. When the section is 381 recommended for renewal, place P36 will receive a token. This place is used to link the 382 gauge module to the ballast module when combined renewal is considered.

\subsection{Modules assembly}

384 The PN models for vertical alignment and gauge described above, can be combined 385 into one model by considering the dependencies resulting from the inspection and the 386 renewal processes. The resulting model is depicted in Figure 12, where place P36 in 387 the gauge module is input to transition T36 in the ballast module. T36 represents the 
event of a combined ballast and sleepers/fastenings renewal driven by sleepers.

389 Indeed, a necessary condition for T36 to fire is that place P36 is marked. This

390 circumstance occurs when sleepers (and consequently also fastenings) are recommended for renewal. This can be due to either sleepers' age, in which case transition T33 fires and adds a token to place P36, or sleepers' conditions, in which case transition T34 fires and adds a token to place P36.

394 FIGURE 12 HERE

\section{Figure 1 PN combining ballast and gauge models.}

\section{$397 \quad 2.5$ Model analysis}

398 The PN models presented in this work contain several non-conventional features that cannot be accommodated by software commercially available for the construction and execution of PN models. Therefore, a bespoke programme have been developed in a

$401 \mathrm{C}++$ environment, that accounts for the additional features introduced in the models.

402 The behaviour of the track system over time is intrinsically stochastic, thus simulation 403 via the Monte Carlo method is the most suitable analysis technique. The Monte Carlo 404 method consists of running a number of simulations duplicating the system behaviour.

405 This process can be seen as a statistical experiment where each simulation is one observation of the system. This approach requires the knowledge of the distributions of

407 times of occurrence of all the significant events which determine the evolution of the 408 system state over time (transitions). For each stochastic transition, the firing time is 409 sampled from the associated stochastic distribution.

410 Here, 2-parameter Weibull distributions are associated to stochastic transitions

411 representing components degradation or failure, while lognormal distribution is 412 generally used for the distribution of times to schedule and perform maintenance. The 413 2-parameter Weibull cumulative distribution function is given by Equation 1.

$414 \quad F(t)=1-e^{-\left(\frac{t}{\eta}\right)^{\beta}}$ 
415 where $\eta$ is the scale parameter and $\beta$ is the shape parameter. The scale parameter is 416 the time at which $63.2 \%$ of the population failed (or degraded to a given state). The 417 shape parameter is indicative of the rate of degradation. Values of $\beta>1$ indicates that 418 the degradation rate increases with time; this is typical of components subject to wear 419 and ageing. A value of $\beta=1$ instead is typical of components exhibiting a constant 420 degradation (or failure) rate. In this last case the Weibull distribution becomes a 421 negative-exponential distribution and the scale parameter represents the mean time to 422 failure of the component.

423 From the cumulative distribution the firing time is evaluated by first generating a 424 random number $X$ uniformly distributed between 0 and 1 , and then equating it to the 425 cumulative probability as in Equation 2,

$426 \quad F(t)=1-e^{-\left(\frac{t}{\eta}\right)^{\beta}}=X$

427 from which the time is obtained as in Equation 3

$428 t=\eta[-\ln X]^{\frac{1}{\beta}}$.

429 Each simulation represents a lifecycle of the track section. During each simulation, the 430 marking of all places and the firing of all transitions of interest in the PN is monitored.

431 This enables the following statistics to be evaluated:

- Number of routine maintenance actions,

- Number/duration/probability of speed restrictions and line closures,

- Probability of being in any of the possible states (good/requiring routine maintenance/requiring emergency intervention), - Average time of section renewal.

\section{Model application}

438 The effects of a range of different maintenance policies on track geometry have been 439 investigated through simulation of the PN model for a number of combinations of the maintenance parameters. Table 1 contains the value of the parameters related to the 
441 inspection frequency $\vartheta_{\mathrm{i}}$, the time to perform routine maintenance and emergency

442 intervention (mean $\mu_{\mathrm{i}}$ and variance $\mathrm{s}_{\mathrm{i}}^{2}$ of the corresponding lognormal distributions) for

443 both vertical alignment and gauge.

444 Table 1. Maintenance parameters.

\begin{tabular}{|c|c|c|c|c|c|c|}
\hline Inspection (days) & \multicolumn{2}{|c|}{$\Theta_{1}=15$} & \multicolumn{2}{|c|}{$\Theta_{2}=30$} & \multicolumn{2}{|c|}{$\Theta_{3}=120$} \\
\hline Routine (days) & $\mu_{\mathrm{rm}, 1}=20$ & ${\mathrm{Srm}, 1^{2}=5}$ & $\mu_{\mathrm{rm}, 2}=45$ & $\mathrm{Srm}, 2^{2}=10$ & $\mu_{\mathrm{rm}, 3}=60$ & $\mathrm{Srm}, 3^{2}=10$ \\
\hline Emergency (days) & $\mu_{\mathrm{em}, 1}=1$ & Sem, $1^{2}=0.25$ & $\mu_{\mathrm{em}, 2}=3$ & $\mathrm{Sem}, 2^{2}=1$ & $\mu_{\mathrm{em}, 3}=7$ & $\mathrm{Sem}, 3^{2}=2$ \\
\hline
\end{tabular}

446 Table 2 below specifies the thresholds on the SD of the vertical alignment triggering

447 opportunistic $\left(\sigma_{\mathrm{op}}\right)$, routine $\left(\sigma_{\mathrm{rm}}\right)$, emergency maintenance with speed restriction $\left(\sigma_{\mathrm{sr}}\right)$

448 and emergency maintenance with line closure $\left(\sigma_{\mathrm{lc}}\right)$. For this numerical application it has

449 been assumed that the thresholds for critical state is $\sigma_{\mathrm{cr}}=\sigma_{\mathrm{sr}}$ (in simple terms, the next

450 degraded state after the one requiring routine maintenance, is the state requiring a

451 speed restriction. Transition T3 is therefore immediate.)

452 Table 2. Thresholds of SD for vertical alignment and corresponding parameters of 453 Weibull distributions associated to transitions.

\begin{tabular}{c|cc|c|c|c}
\hline \multirow{2}{*}{ SD } & & $\sigma_{\mathrm{op}}$ & $\sigma_{\mathrm{rm}}$ & $\sigma_{\mathrm{sr}}$ & $\sigma_{\mathrm{lc}}$ \\
\cline { 2 - 6 } thresholds & $(1)$ & 3.5 & 4.25 & 4.5 & 5 \\
& $(2)$ & 3.5 & 3.75 & 4.5 & 5 \\
\hline Transitions & & $\mathrm{T} 1$ and T6 & $\mathrm{T} 2$ & $\mathrm{~T} 4$ & $\mathrm{~T} 5$ \\
\hline Weibull & $(1)$ & $\beta=1.4, \eta=1000$ & $\beta=1.55, \eta=300$ & $\beta=1.6, \eta=400$ & $\beta=1.7, \eta=300$ \\
parameters & $(2)$ & $\beta=1.4, \eta=1000$ & $\beta=1.45, \eta=500$ & $\beta=1.6, \eta=500$ & $\beta=1.7, \eta=300$ \\
\hline
\end{tabular}

454

455 In this application two SD levels for routine maintenance $\sigma_{\mathrm{rm}}$ have been considered,

456 namely 4.25 and 3.75, while the other thresholds are left unchanged. Two approaches

457 have been adopted for selecting the SD thresholds. The first pushes the threshold for

458 routine maintenance closer to the limit triggering a speed restriction. The second

459 approach is more conservative, as it establishes a lower threshold for routine

460 interventions. To each pair of consecutive thresholds is associated a distribution of

461 times to degrade from one threshold level to the next. It is assumed that these times

462 are distributed according to a 2-parameter Weibull. Because two different values of $\sigma_{\mathrm{rm}}$

463 have been considered, this means that two different sets of parameters $(\beta, \eta)$ will be 
associated to transitions T2 (from place P2 to place P3) and T3 (from place P3 to place

P4) one for each value of $\sigma_{\mathrm{rm}}$. The Weibull parameters for transitions T1 to T6 are also given in Table 2.

467 Concerning the gauge, in this application it is assumed that the number of consecutive failed elements (sleepers and/or fastenings) that trigger a line closure and a speed restriction is 10 and 8 sleepers and/or pairs of fastenings respectively. From the definition of a cluster size as given in section 2.3 follows that each cluster contains 10 sleepers and 20 fastenings. While the thresholds on the number of consecutive failed elements for speed restriction and line closure are kept unchanged, the number of failed elements triggering a routine intervention, $\mathrm{N}_{\mathrm{f}, \mathrm{min}}$, is varied. Specifically, three different $\mathrm{N}_{t, \min }$ values have been analysed, i.e. 2, 4 and 5. An opportunistic intervention is possible if one or more $\left(<\mathrm{N}_{\mathrm{f}, \min }\right)$ components fail. Speed restrictions and line closures are followed by emergency and immediate interventions respectively. It is assumed

477 here that the lifetimes of the individual sleeper and fastenings follow a 2-parameter Weibull distribution with parameters given in Table 3.

Table 3. Weibull parameters for sleepers and fastenings lifetime.

\begin{tabular}{c|l}
\hline Concrete sleepers & $\beta=1.4, \eta=9125$ \\
\hline Fastenings & $\beta=1.2, \eta=3650$ \\
\hline
\end{tabular}

480

481 The combinations of the maintenance parameters in Tables 1 with the two thresholds 482 used for $\sigma_{\mathrm{rm}}$ (Table 2) result in 54 strategies for maintaining the vertical alignment (C1 483 to C54); these are detailed in Table 4. The parameters in Table 1, combined with the 484 three values of $\mathrm{N}_{\mathrm{f}, \mathrm{min}}$ determine 81 strategies for gauge maintenance (S1 to S81) as 485 shown in Table 5. It is assumed that the ballast is renewed as soon as five out of eight unit sections $\left(1 / 8^{\text {th }}\right.$ mile $)$ every mile of track has been tamped 15 times $\left(N_{\max , t a m p}=15\right)$.

487 Sleepers are renewed as soon as they reach their lifetime which, in this example is assumed to be 35 years. It is also assumed that ballast, sleepers and fastening all start from new conditions. 
Table 4 Strategies for maintaining the vertical alignment.

\begin{tabular}{|c|c|c|c|c|c|c|c|c|c|c|c|c|c|}
\hline \multirow[b]{2}{*}{ ID } & \multicolumn{6}{|c|}{$\sigma_{\mathrm{rm} 1}=4.25$} & \multirow[b]{2}{*}{ ID } & \multicolumn{6}{|c|}{$\sigma_{\mathrm{rm} 2}=3.75$} \\
\hline & $\theta$ & $\mu_{\mathrm{rm}}$ & $S_{r_{m}}^{2}$ & $\mu_{\mathrm{em}}$ & $S_{e m^{2}}$ & $\mathbf{N}_{\text {max,tamp }}$ & & $\theta$ & $\mu_{\mathrm{rm}}$ & $S_{\mathrm{rm}^{2}}{ }^{2}$ & $\mu_{\mathrm{em}}$ & $S_{e m^{2}}$ & $\mathbf{N}_{\text {max,tamp }}$ \\
\hline $\mathrm{C} 1$ & 15 & 20 & 5 & 1 & 0.25 & 15 & C28 & 15 & 20 & 5 & 1 & 0.25 & 15 \\
\hline $\mathrm{C} 2$ & 15 & 45 & 10 & 1 & 0.25 & 15 & C29 & 15 & 45 & 10 & 1 & 0.25 & 15 \\
\hline C3 & 15 & 60 & 10 & 1 & 0.25 & 15 & C30 & 15 & 60 & 10 & 1 & 0.25 & 15 \\
\hline C4 & 15 & 20 & 5 & 3 & 1 & 15 & C31 & 15 & 20 & 5 & 3 & 1 & 15 \\
\hline C5 & 15 & 45 & 10 & 3 & 1 & 15 & C32 & 15 & 45 & 10 & 3 & 1 & 15 \\
\hline C6 & 15 & 60 & 10 & 3 & 1 & 15 & C33 & 15 & 60 & 10 & 3 & 1 & 15 \\
\hline $\mathrm{C} 7$ & 15 & 20 & 5 & 7 & 2 & 15 & C34 & 15 & 20 & 5 & 7 & 2 & 15 \\
\hline C8 & 15 & 45 & 10 & 7 & 2 & 15 & C35 & 15 & 45 & 10 & 7 & 2 & 15 \\
\hline C9 & 15 & 60 & 10 & 7 & 2 & 15 & C36 & 15 & 60 & 10 & 7 & 2 & 15 \\
\hline $\begin{array}{c}\text { C10 } \\
\text { to } \\
\text { C18 }\end{array}$ & 30 & \multicolumn{5}{|c|}{ Same as $C 1$ to $C 9$} & $\begin{array}{c}\text { C37 } \\
\text { to } \\
\text { C45 }\end{array}$ & 30 & \multicolumn{5}{|c|}{ Same as $\mathrm{C} 28$ to $\mathrm{C} 36$} \\
\hline $\begin{array}{c}\text { C19 } \\
\text { to } \\
\text { C27 }\end{array}$ & 120 & \multicolumn{5}{|c|}{ Same as $C 1$ to $C 9$} & $\begin{array}{c}\text { C46 } \\
\text { to } \\
\text { C54 }\end{array}$ & 120 & \multicolumn{5}{|c|}{ Same as C28 to C36 } \\
\hline
\end{tabular}

Table 5 Strategies for maintaining the gauge.

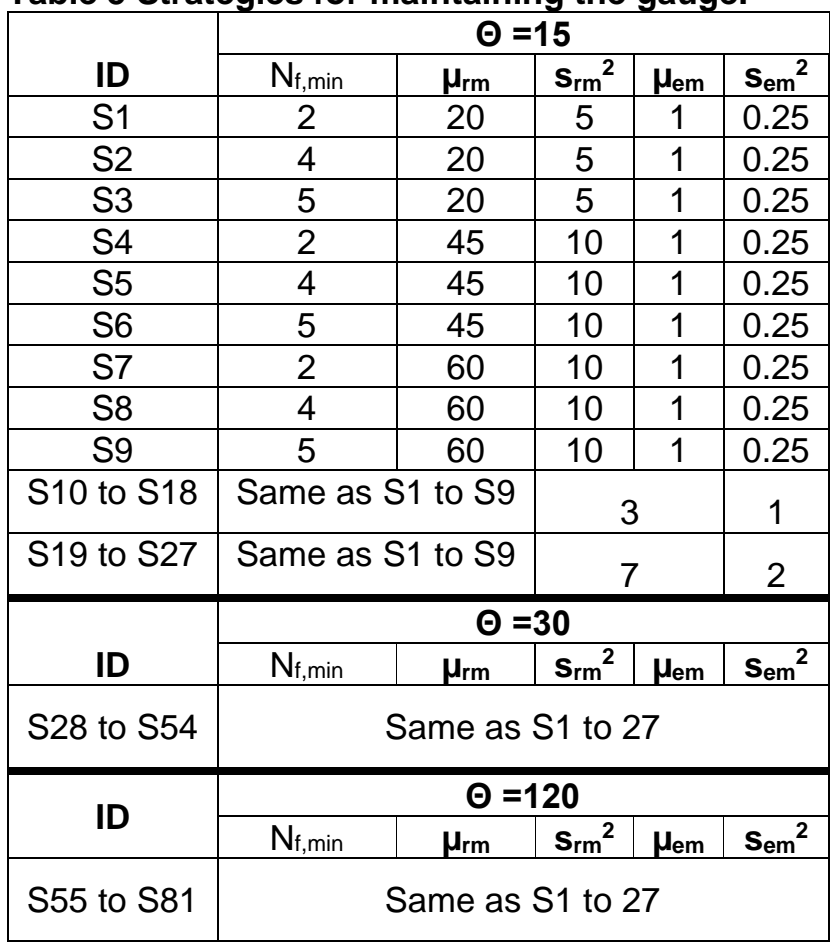

493

\section{$494 \quad 3.1$ Results}

495 Convergence of results is reached after 500 simulations. In the following, simulation

496 results showing the effects on both track gauge and vertical alignment are presented

497 and discussed. Figures are given per mile of track, under the assumption of

498 homogeneous characteristics, and provide average values over the entire simulated

499 time. The simulated time varies as it depends on when renewal is required (a 
simulation is stopped when the section is recommended for renewal). The

501 computational time required to simulate all considered strategies, each 500 times is

502 about 10 minutes. Figures 13 to 17 show the probability of being in good conditions

503 (Figure 13), of a speed restriction being imposed (Figure 14), the average number of

504 routine interventions (Figure 15) and opportunistic interventions (Figure 16), and the

505 renewal times (Figure 17) for each maintenance strategy. The combination of the

506 maintenance parameters corresponding to each strategy is also specified in each

507 figure.

508 FIGURES 13, 14, 15, 16, 17 HERE.

509

510

Figure 2 Probability of good state (ballast)

511

Figure 3 Probability of speed restriction imposed due to ballast degradation.

Figure 4 Number of routine interventions on ballast.

516 Figure 5 Number of opportunistic interventions on ballast.

Figure 6 Ballast renewal times in days.

519

Results show that the parameter with a major influence on the asset performance is the threshold $\sigma_{\mathrm{rm}}$ triggering routine maintenance, followed by the inspection frequency and the mean time to perform routine maintenance. The probability of being in good conditions is generally higher for lower thresholds $\sigma_{\mathrm{rm}}$, and decreases with increasing

524 mean time to perform routine maintenance, while the probability of a speed restriction shows a complete opposite trend. This is because the longer it takes to perform routine maintenance, and the higher it is the likelihood that conditions will deteriorate further to a level requiring a speed restriction. Such condition, however, is only discovered

528 through inspection; clearly the longer the inspection period, the less the probability of 529 actually revealing such a state. The number of routine interventions is higher for lower 
530 thresholds $\sigma_{\mathrm{rm}, 2}$, meaning also higher intervention costs. The renewal times are mainly

531 affected by the threshold $\sigma_{\mathrm{rm}}$; higher $\sigma_{\mathrm{rm}}$ values determine higher renewal times. This is 532 mainly due to the fact that the ballast degradation rate increases with the number of 533 tamps performed, which is higher for lower thresholds $\sigma_{\mathrm{rm}}$, as also shown in Figure 13.

534 The renewal times obtained for $\sigma_{\mathrm{rm}, 1}$ are in the range between 12490 and 13575 days, 535 namely between 34,23 and 37,2 years, and always below 13000 days (35,6 years) for 536 inspection period of 15 and 30 days. For the more conservative threshold $\sigma_{\mathrm{rm}, 2}$ the 537 renewal times lay between 9275 and 10225 days (25,4-28 years), and always below 5389750 days (26,7 years) for inspection period up to 30 days. The higher threshold $\sigma_{\mathrm{rm}, 1}$, 539 if combined with a more frequent inspection and a quicker response to the need for 540 maintenance, enable longer ballast lifetime to be achieved. Given that the sleepers average lifetime is approximately 35 years, this also allows for ballast and sleepers

542 renewal to be combined without any loss of sleepers useful life, or considerably

543 frequent need for ballast tamping.

544 Figures 18, 19 and 20 represents the probability of being in a state requiring routine 545 maintenance, emergency intervention with a speed restriction and immediate 546 intervention with line closure respectively, resulting from the implementation of each 547 strategy.

548 FIGURES 18, 19, 20 HERE

Figure 7 Probability of being in a state where gauge requires routine maintenance. Figure 8 Probability of speed restrictions due to gauge degradation.

Figure 20 Probability of line closure due to gauge degradation.

556 Results are clustered into three main groups based on the minimum number of 557 components $N_{f, \text { min }}$ triggering a routine intervention. Parameter $N_{f, \min }$ appears to play a

558 fundamental role in determining the gauge response. The probability of requiring a 
routine intervention decreases with $\mathrm{N}_{\mathrm{f}, \text { min }}$ while the probability of requiring an

560 emergency intervention given that a speed restriction or a line closure are imposed,

561 increases. This trend can be explained by observing that the higher the threshold

562 triggering routine maintenance, the higher is the chance that additional components,

563 either sleepers or fastenings, will fail before a routine intervention is performed, thus

564 causing the gauge to spread to a level requiring a speed restriction. Second to $N_{f, m i n}$,

565 the inspection frequency affect the gauge response, with its influence being more

566 evident for the probability of a line closure, especially for higher values of $\mathrm{N}_{\mathrm{f}, \mathrm{min}}$. This is

567 because the worst the conditions, the faster the degradation.

568 Figure 21 shows the total number of sleepers replaced, while the number of fastenings

569 replaced is given in Figure 22. Figure 23 depicts the number of grouped interventions

570 involving sleepers and fastenings within multiple adjacent sections. Grouped

571 interventions means that if a routine intervention is carried out for the elements within a

572 cluster to restore the correct gauge, then ineffective sleepers and fastenings are also

573 replaced within adjacent clusters where routine intervention has been scheduled for a

574 later date, or opportunistic intervention is suitable. This allows taking advantage of the

575 track possession. As expected, the lower the threshold $\mathrm{N}_{\mathrm{f}, \min }$, the higher the number of

576 sleepers replacement.

577 FIGURE 21 HERE

578

579 Figure 21 Total number of sleepers replaced.

580

581 FIGURE 22 HERE

582 Figure 9 Total number of fastenings replaced.

583

584 FIGURE 23 HERE

585 Figure 10 Total number of grouped interventions (multiple sleepers and fastenings

586 replacement).

587 
588 A different behaviour is instead observed for grouped interventions. The highest

589 number of grouped interventions is always obtained for $N_{f, \min }=4$ and inspection

590 frequencies of 15 and 30 days, regardless of the other maintenance parameters.

591 Indeed, it will take longer for a section to enter a state requiring routine maintenance if

$592 \mathrm{~N}_{\mathrm{f}, \min }=4$ than if $\mathrm{N}_{\mathrm{f}, \min }=2$. This means that, before a section will need a routine

593 intervention, it will be more likely that sleepers and fastenings in other locations along

594 the line will have also failed if $\mathrm{N}_{\mathrm{f}, \min }=4$ than if $\mathrm{N}_{\mathrm{t}, \min }=2$. However, if the threshold

595 triggering routine maintenance is pushed closer to the one triggering an emergency

596 interventions, as for $N_{f, \min }=5$, then it is more likely that the section currently scheduled

597 for a routine maintenance will degrade further to a state requiring an emergency action

598 before a failure occur in any other location along the line. This observation is also

599 supported by the fact that the probability of being in a state requiring a speed restriction

600 and emergency intervention takes the higher value when $N_{f, \min }=5$ as shown in Figure

601 16. If inspection intervals increase to 120 days, then it will take longer for the need for

602 routine maintenance to be revealed. Therefore, when $\mathrm{N}_{\mathrm{f}, \mathrm{min}}=4$, the section is more

603 likely to degrade to a state requiring a speed restriction before inspection is performed.

604 On the other hand, when $\mathrm{N}_{\mathrm{t}, \min }=2$, longer inspection intervals means that failures in

605 other locations along the line might occur before inspection is performed.

\section{4. Conclusions}

607 In this paper a simulation tool based on Petri nets has been presented, which models

608 track geometry degradation and the corresponding maintenance actions that can be

609 performed. The model accounts for vertical alignment variations and gauge spreading

610 due to ballast and sleepers/fastening failures respectively. The model enables the track

611 geometry conditions, probability of failure modes leading to speed restrictions and line

612 closures, and the number of interventions performed during a given time horizon to be

613 predicted for a wide range of maintenance strategies. Along with the probability of

614 speed restrictions and line closures being imposed, also the average number and 
615 duration of such restrictive measures can be recorded during simulations. These

616 provide indirect indication of the impact that different maintenance strategies will have

617 on service if implemented. Although the proposed model does not directly quantify the

618 delays and corresponding costs, it enables a comparison between different strategies

619 to be drawn, based on the number of interventions (routine, opportunistic and

620 emergency) and the unavailability of the track due to unplanned speed restrictions and

621 line closures. Clearly, an actual evaluation of more detailed service performance

622 measures which account for the actual delays and/or journey cancellations, will require

623 the use of specific software that model the interactions between train services and

624 infrastructure failures and maintenance such as OpenTrack, RailSys and TRAIL. The

625 results obtained from the proposed model can be used within the aforementioned

626 software to generate disruption scenarios that are directly linked to a given

627 maintenance strategy. The model can also be used to gain insight into the potential

628 effects of new maintenance strategies on the asset performance thus partly

629 compensating for the lack of real data whose collection would require years if not

630 decades.

631 Acknowledgements

632 John Andrews is the Network Rail Professor of Infrastructure Asset Management and

633 Director of Lloyd's Register Foundation (LRF) (Lloyd's Register Foundation supports

634 the advancement of engineering-related education, and funds research and

635 development that enhances safety of life at sea, on land and in the air) Resilience

636 Engineering Research Group at the University of Nottingham. The authors gratefully

637 acknowledge the support of these organizations.

References

639

640

Burkhalter, M., Martani, C., \& Adey, B. (2018). Determination of risk-reducing intervention programs for railway lines and the significance of simplification.

641 Journal of Infrastructure Systems, 24(1): 04017038. 
642 Moreu, F., Spencer Jr., B., Foutch, D., \& Scola, S. (2017). Consequence-based management of bridge networks. Structure and Infrastructure Engineering, $13: 2,273-286$.

645

646

\section{References}

648 Andrews J. (2012) A modelling approach to railway track asset management. Proceedings of

649 the Institution of Mechanical Engineers, Part F: Journal of Rail and Rapid Transit, 227(1):56-

$650 \quad 73$

651 Andrews J., Prescott D., and De Rozieres F. (2014) A stochastic model for railway track asset 652 management. Reliability Enginering and System Safety, 130:76-84.

653 Bai L., Liu R., Sun Q., Wang F., and Xu P. (2013) Markov-based model for the prediction of railway track irregularities. Proceeding of the Institution of Mechanical Engineers, Part F: J Rail Rapid Transit, 229(2):150-159.

656

David R. and Alla H. (2010) Discrete, continuous and hybrid Petri nets. Springer Science \& 657 Business Media.

658 Lake M., Ferreira L., and Murray M. (2000a) Cost-benefit analysis of sleeper replacement 659 strategies: simulation model. Transportation Research Record: Journal of the Transportation 660 Research Board 1713, pages 15-21.

661 Lake M., Ferreira L., and Murray M. (2000b) Using simulation to evaluate rail sleeper 662 replacement alternatives. Transportation Research Record 1785, pages 58-63.

663 Meier-Hirmer C., Riboulet G., and Sourget F. (2009) Maintenance optimization for a system with 664 a gamma deterioration process and intervention delay: application to track maintenance.

665 Proceeding of the Institution of Mechanical Engineers, Part O: J Risk and Reliability, $666 \quad 223: 189-198$.

667 Murata T. (1989) Petri nets: properties, analysis and applications. Proceedings of the $668 \quad$ IEEE,77(4):541-580.

669 Prescott D. and Andrews J. (2013a) Investigating railway track asset management using a 670 Markov analysis. Proceeding of the Institution of Mechanical Engineers, Part F: J Rail Rapid 671 Transit, 229(4):402-416. 
Prescott D. and Andrews J. (2013b) A track ballast maintenance and inspection model for a rail network. Proceeding of the Institution of Mechanical Engineers, Part O: J Risk and Reliability, 227(3):251-266.

675 Yun W.Y. and Ferreira L. (2003) Prediction of the demand of the railway sleepers - a simulation 676 model for replacement strategies. International Journal of Production Economics, pp 589595.

678 Zhao J., Chan A.H.C., and Burrow M.P.N. (2007) Reliability analysis and maintenance decision for railway sleeper using track condition information. The Journal of the Operational

680 Research Society, 58(8):1047-1055.

681

OpenTrack. OpenTrack Railway Technology Ltd. Gubelstr. 28, CH 8050 Zurich, Switzerland.

682

RailSys. Rail Management Consultants GmbH. Luetzerodestrasse 10, 30161 Hannover,

683 Germany.

684

685

FIGURES

686

687
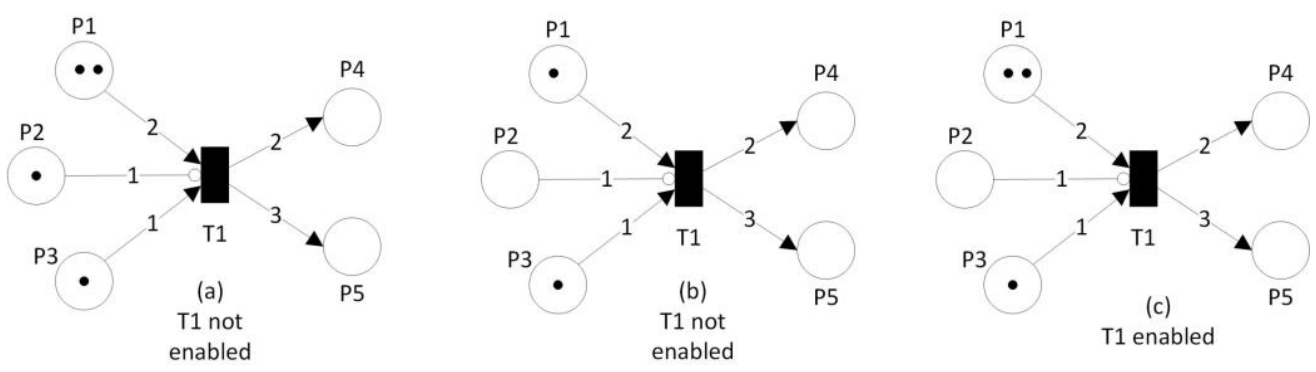

688
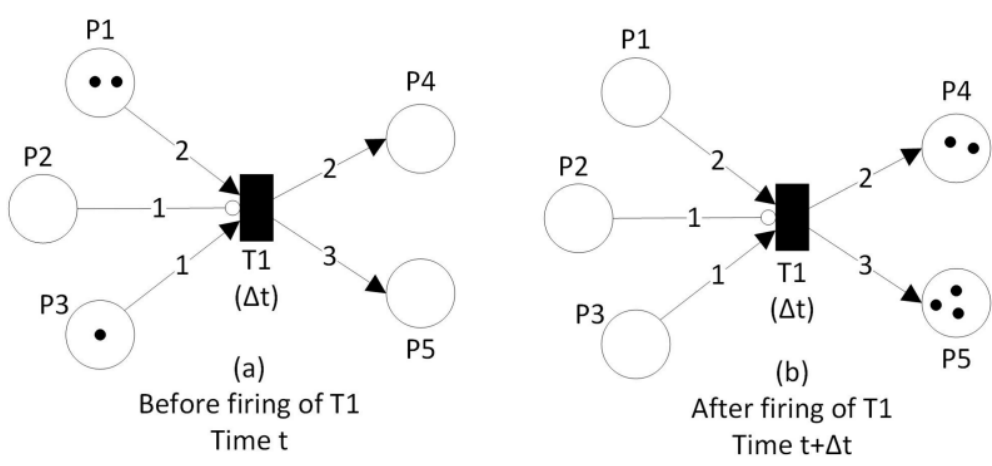

689

690 


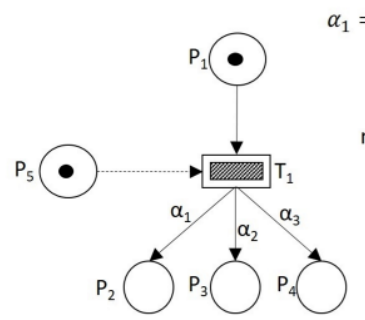

(Before firing)

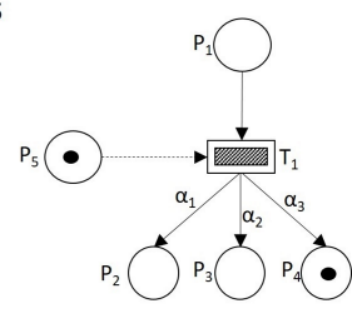

(After firing)

\begin{tabular}{|c|c|c|c|}
\hline \multicolumn{2}{|c|}{$\begin{array}{l}\text { Transitions with standard } \\
\text { firing mode }\end{array}$} & \multicolumn{2}{|c|}{$\begin{array}{l}\text { Transitions with non-standard } \\
\text { firing mode }\end{array}$} \\
\hline & Immediate transition & & Immediate transition \\
\hline $\mathrm{t}$ & $\begin{array}{l}\text { Timed transition } \\
\text { (deterministic or stocastic) }\end{array}$ & $\mathrm{t}$ & $\begin{array}{l}\text { Timed transition } \\
\text { (deterministic or stocastic) }\end{array}$ \\
\hline t) & $\begin{array}{l}\text { Timed transition with } \\
\text { associated set of firing } \\
\text { time distributions }\end{array}$ & $t$ & $\begin{array}{l}\text { Timed transition with } \\
\text { associated set of firing } \\
\text { time distributions }\end{array}$ \\
\hline
\end{tabular}

694

695

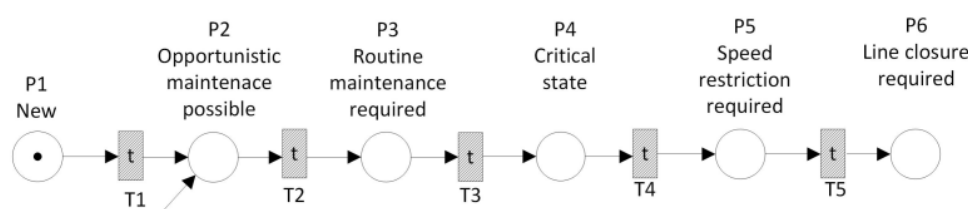

696

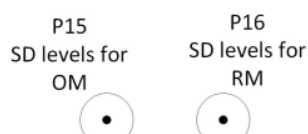

697
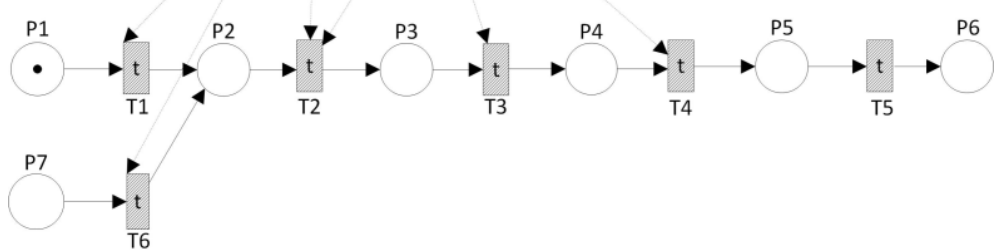


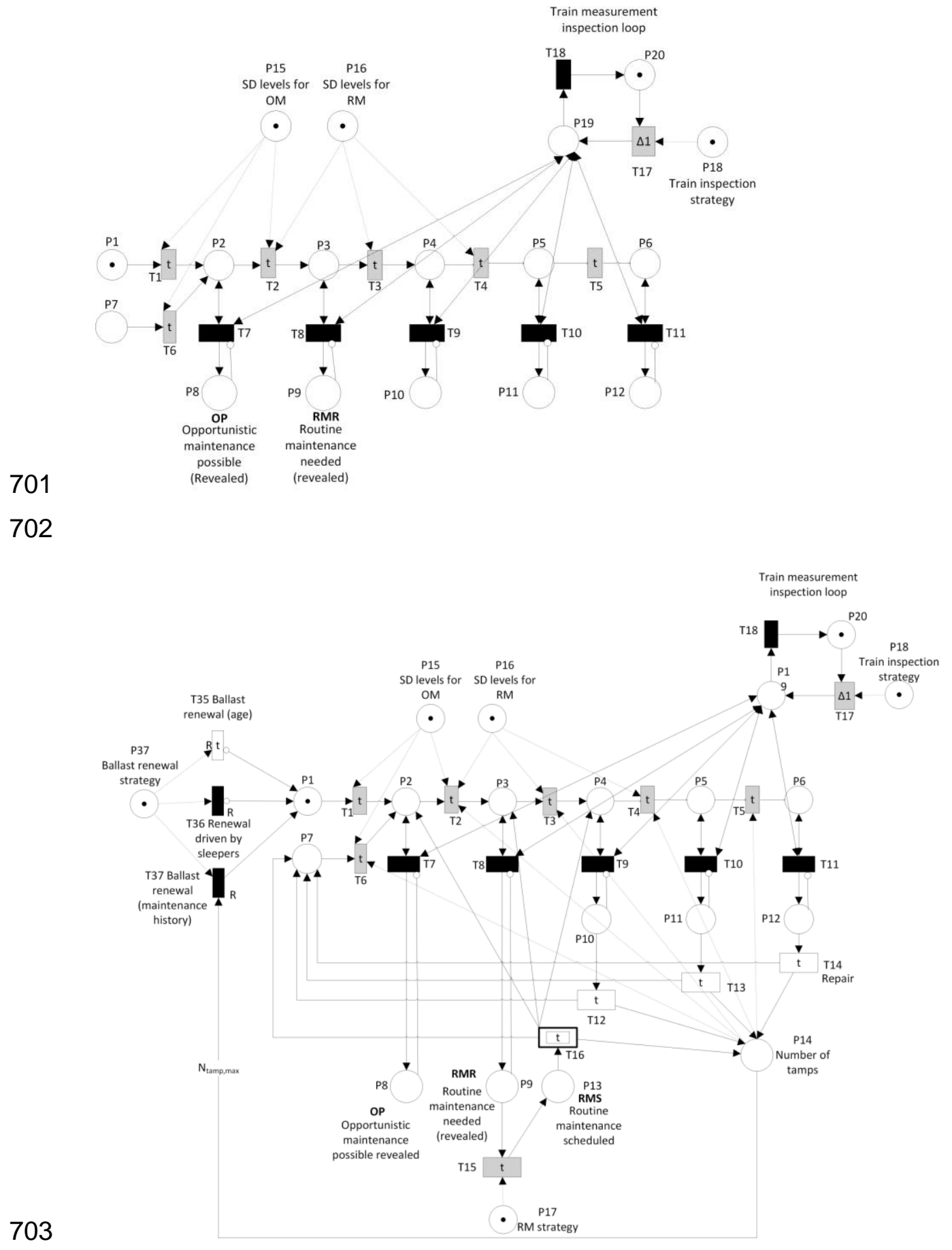

704

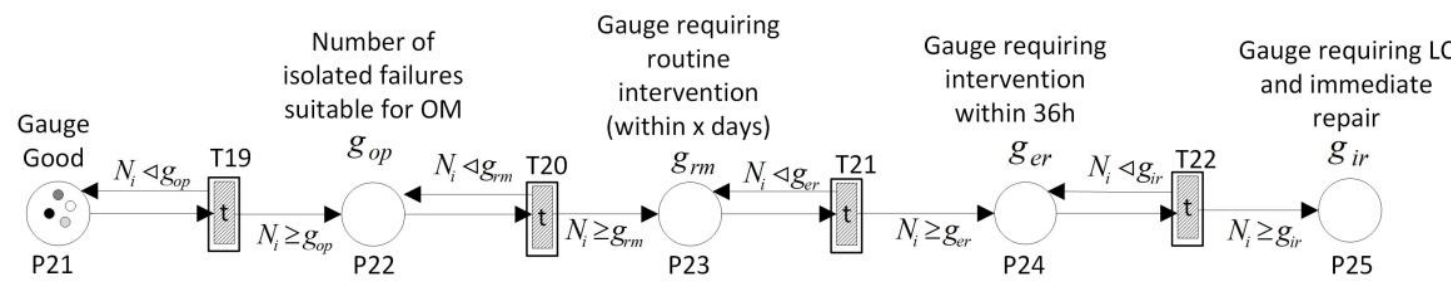




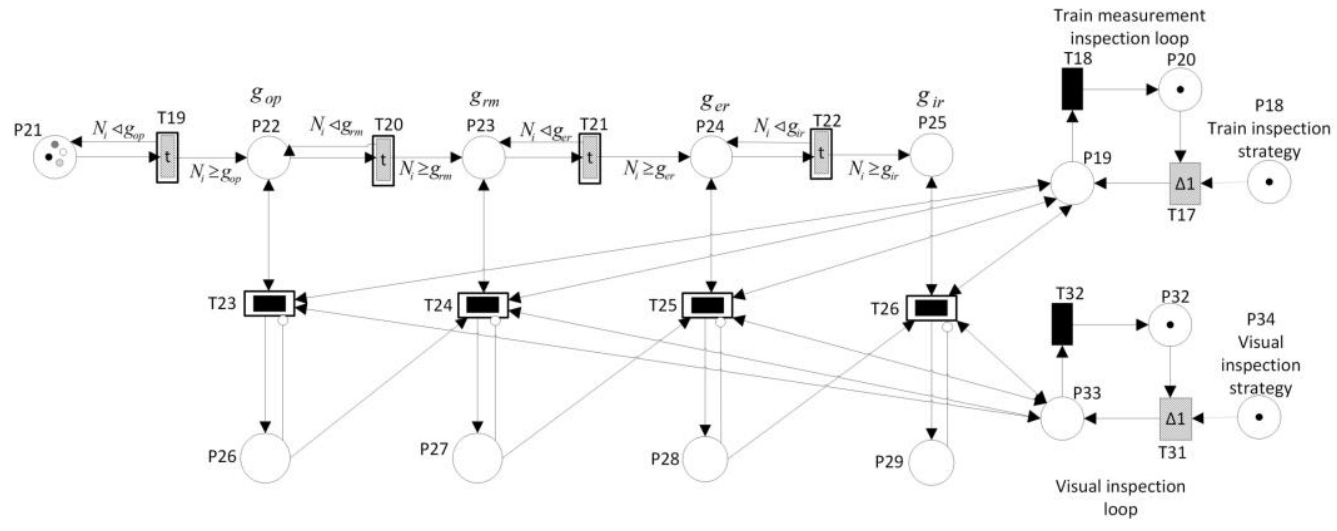

708

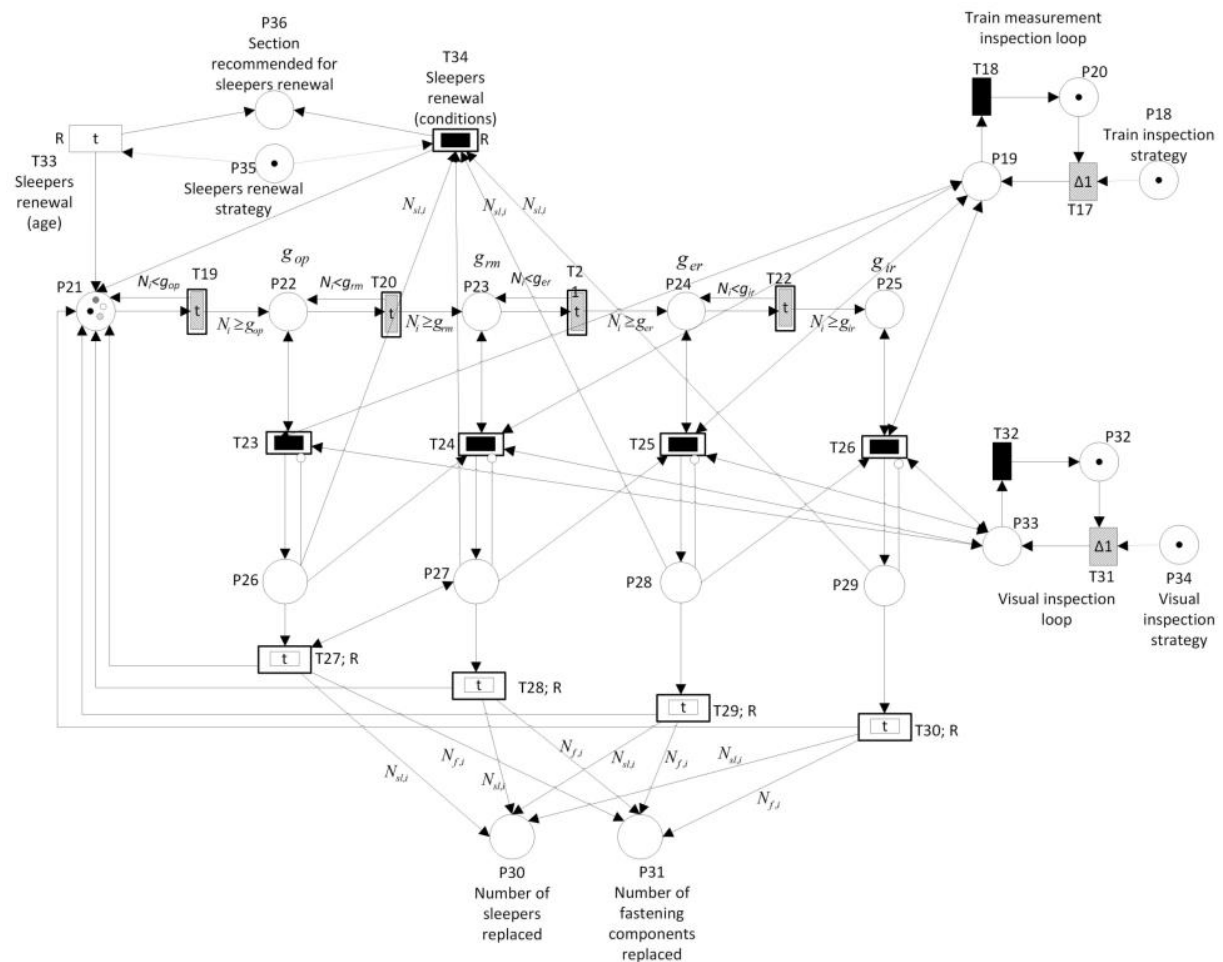

710 


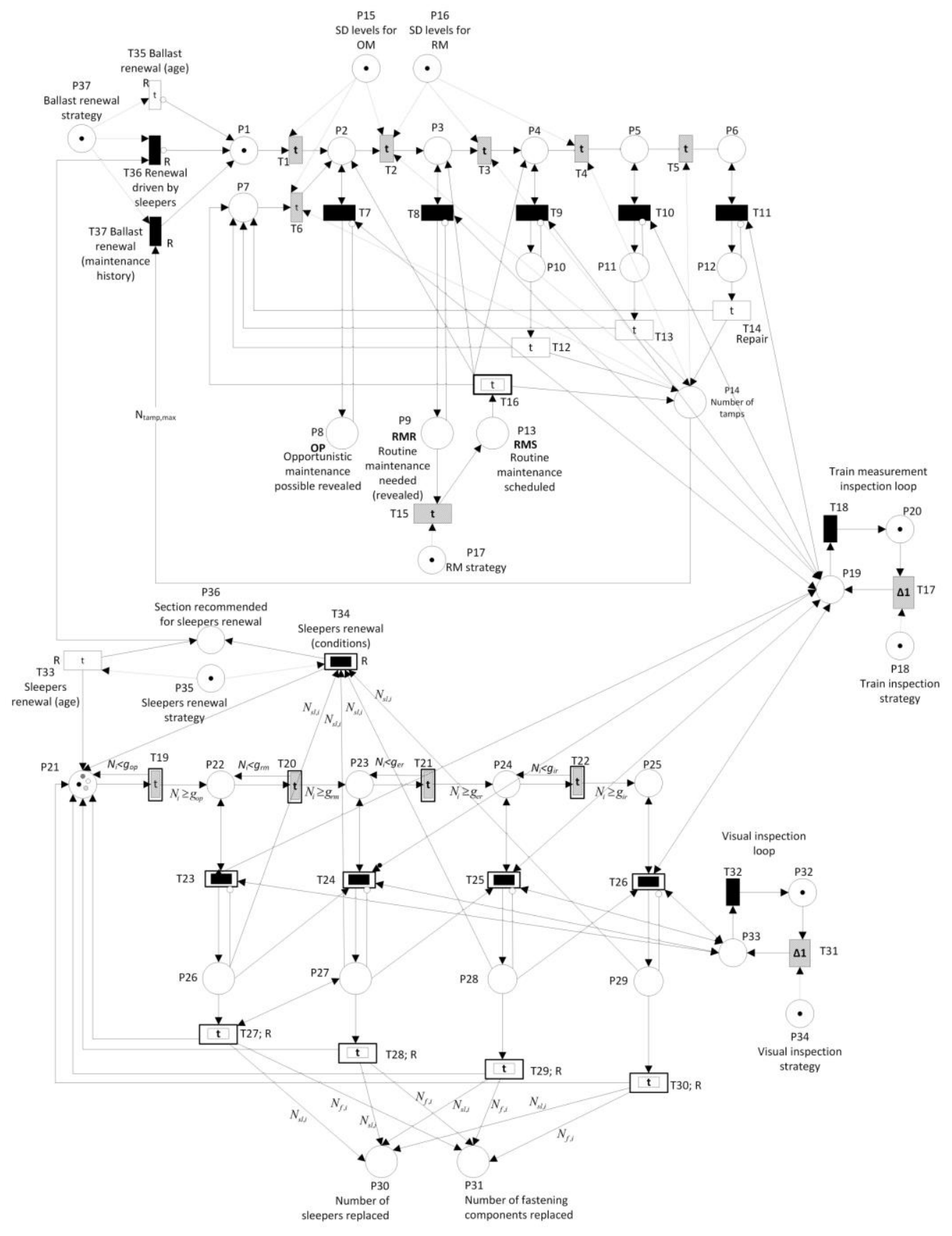

712

713 


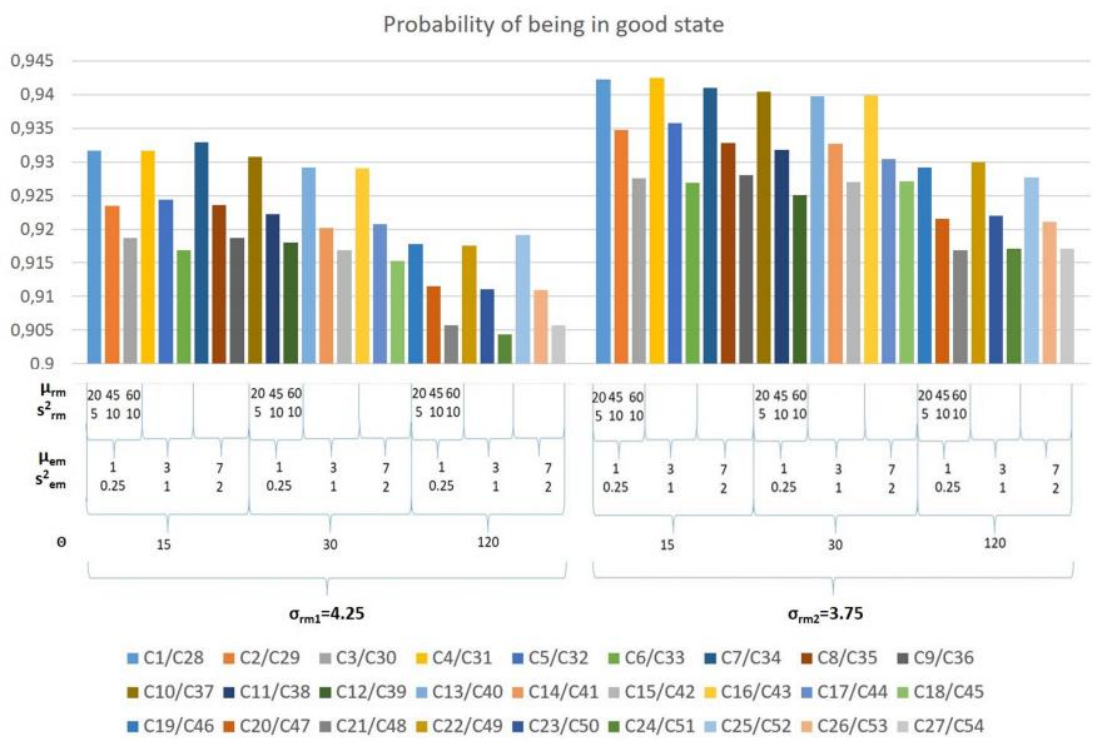

715
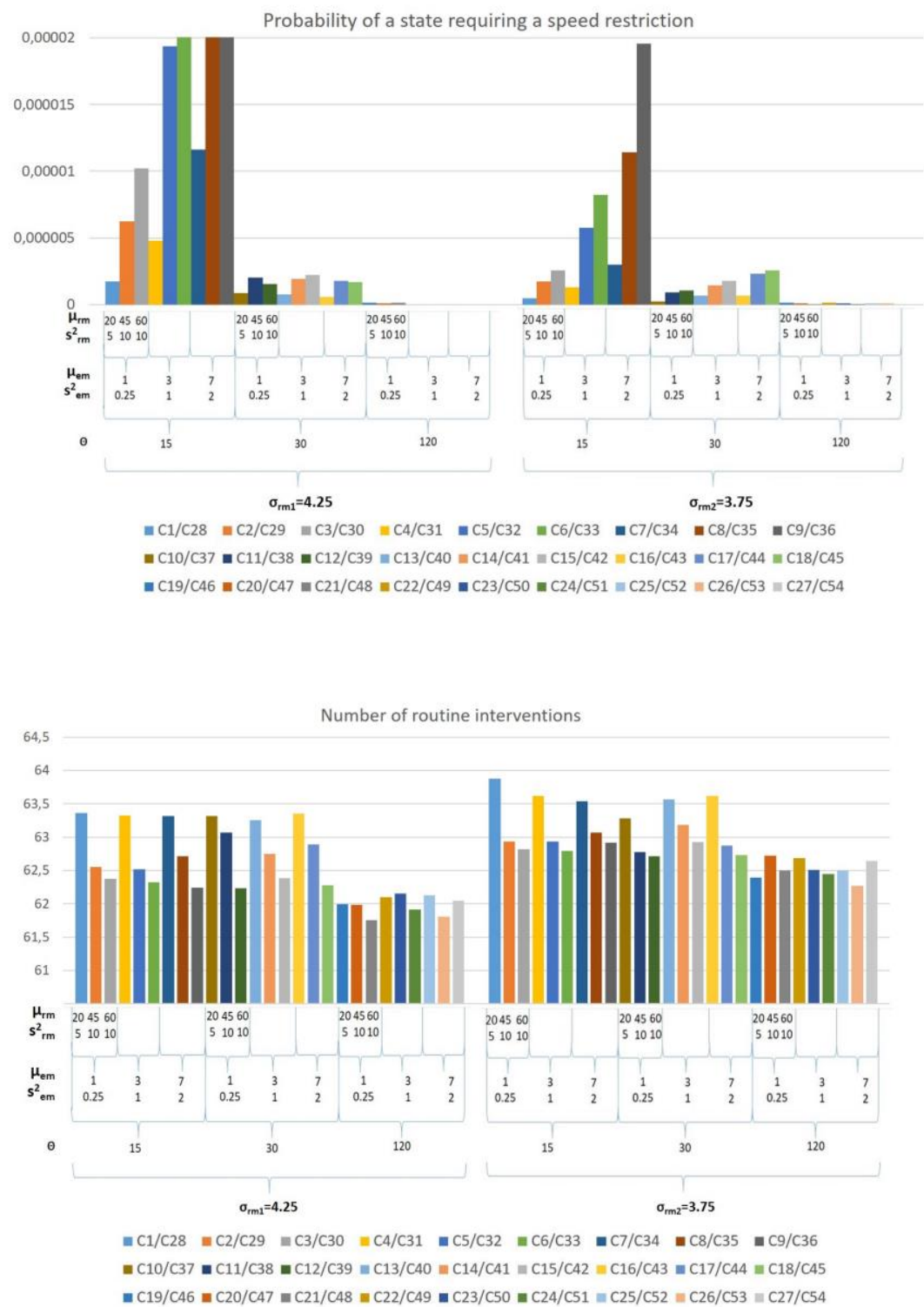


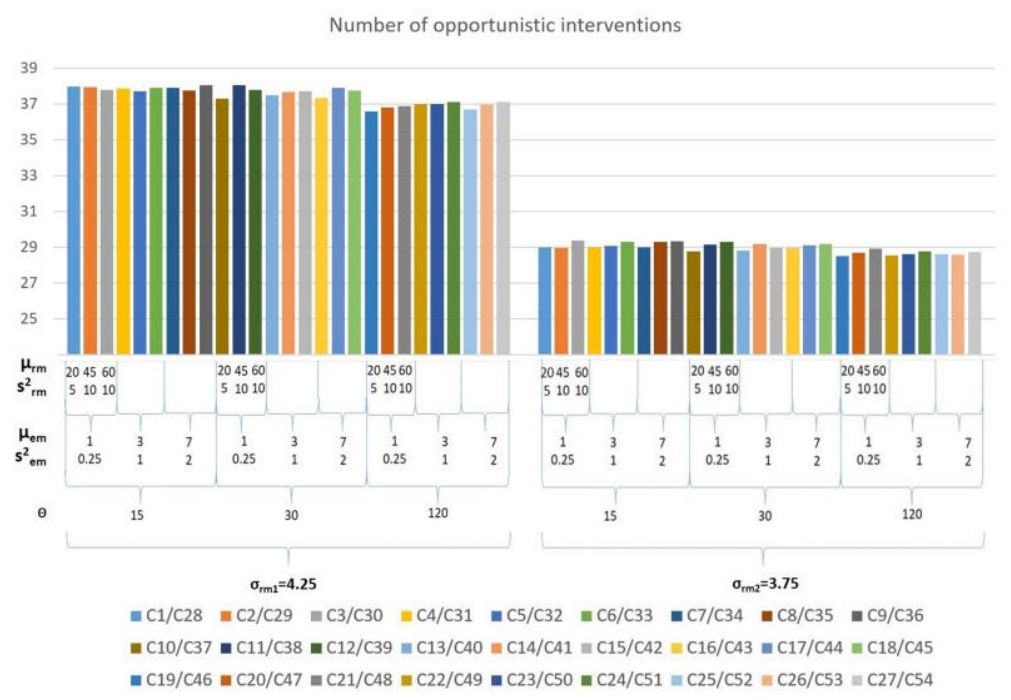

721

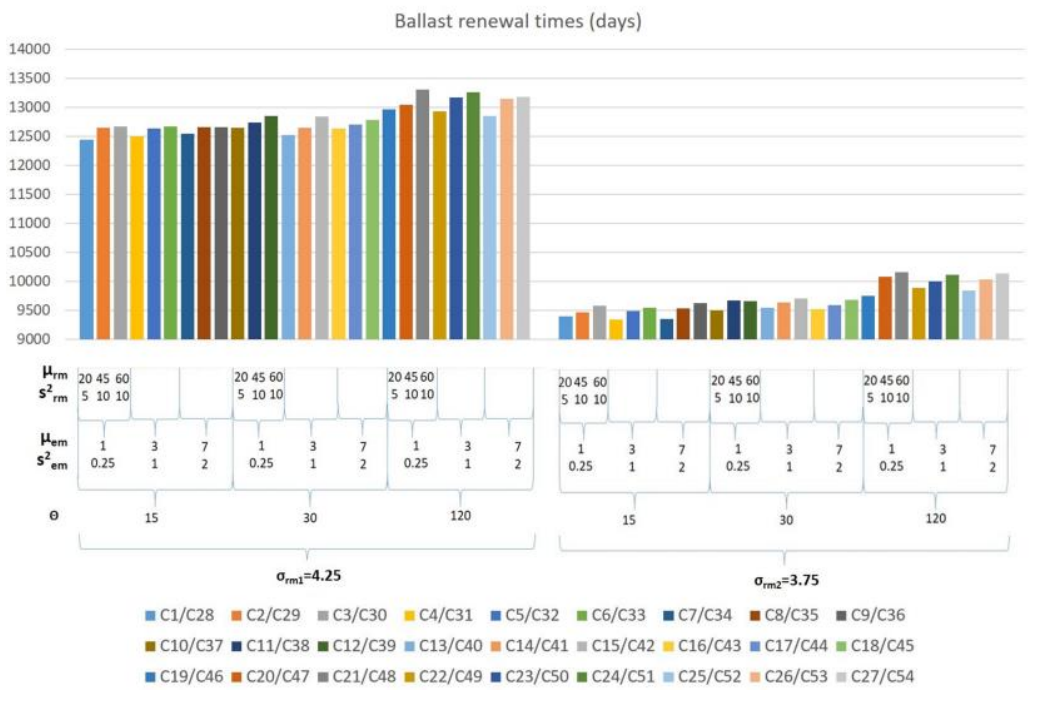

723

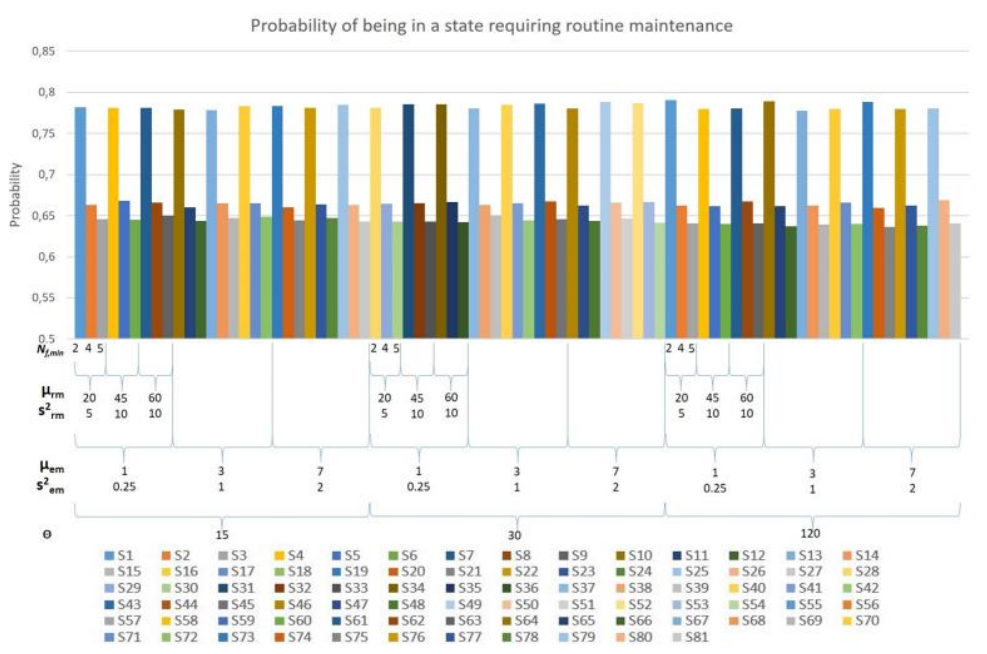




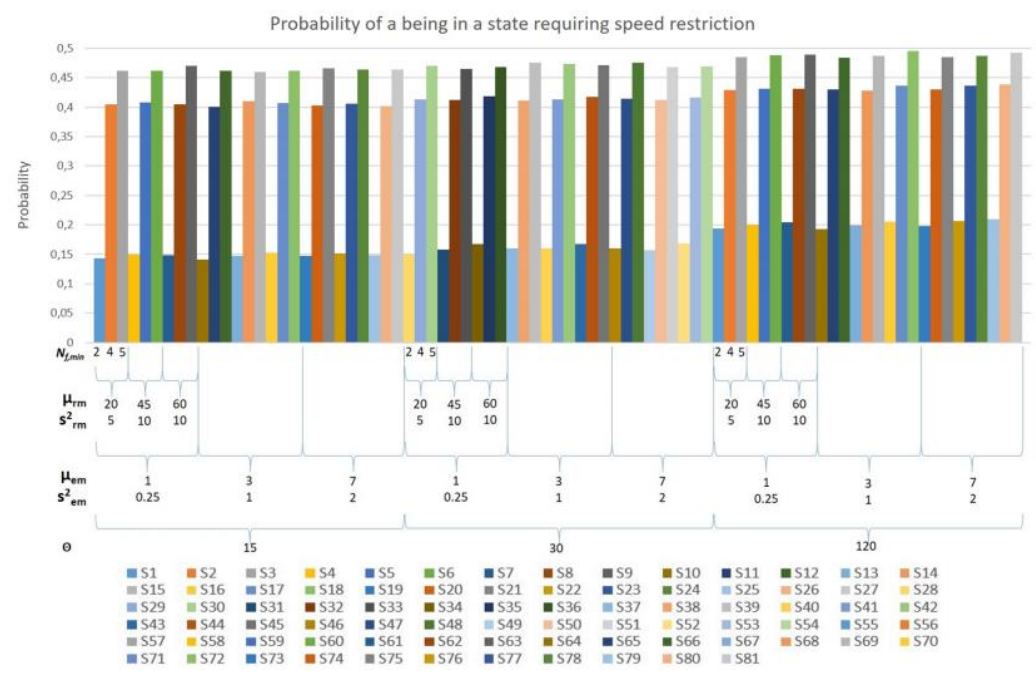

727

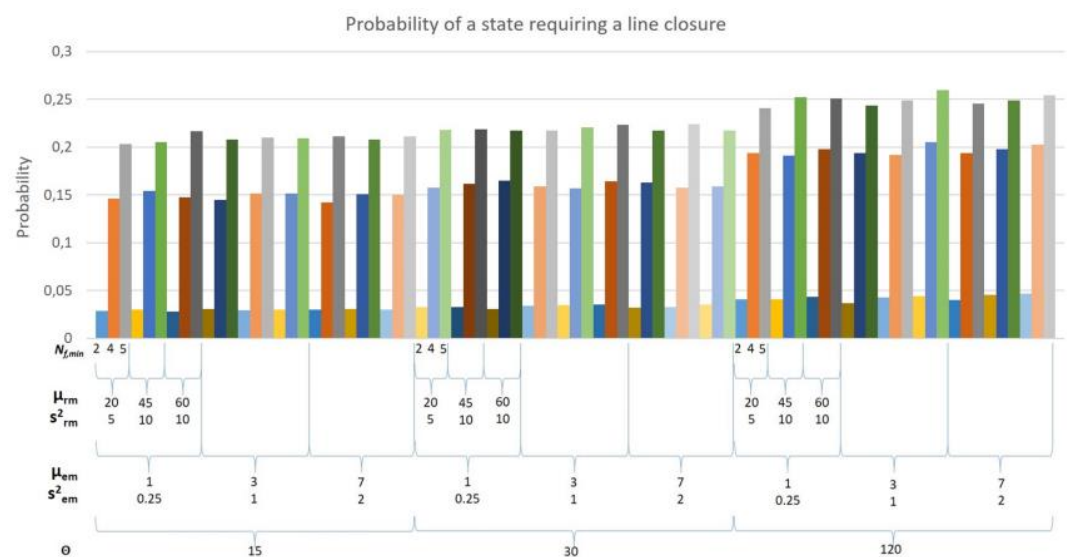

729

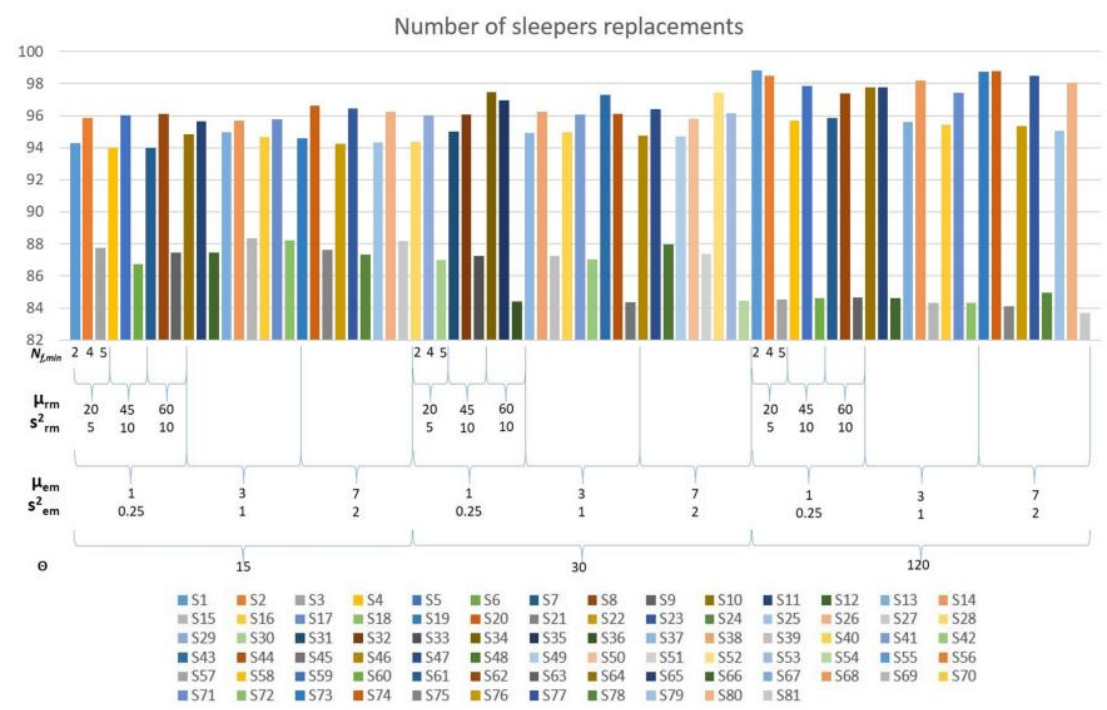




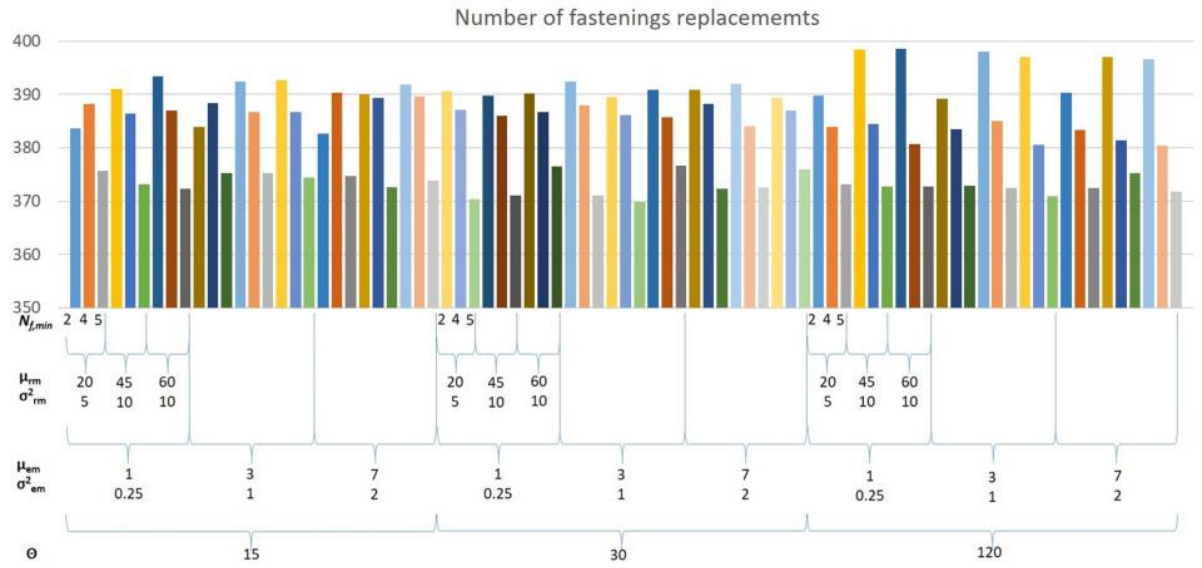

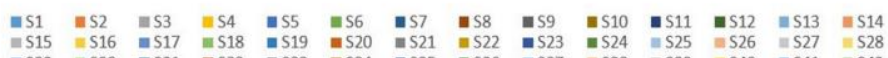

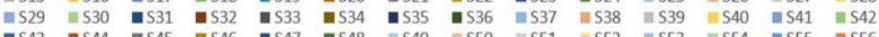

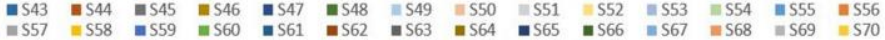

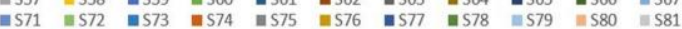

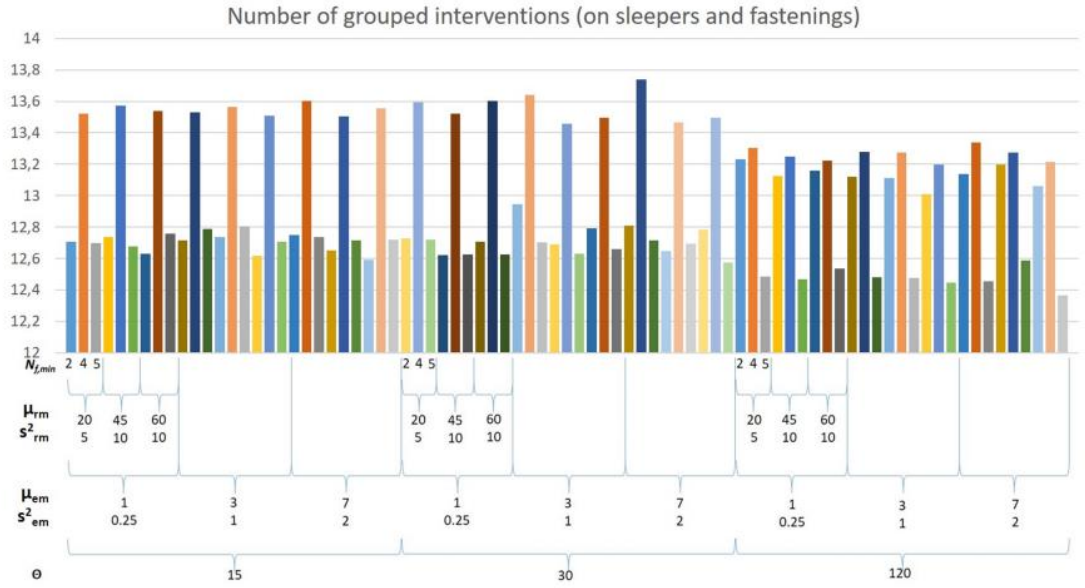

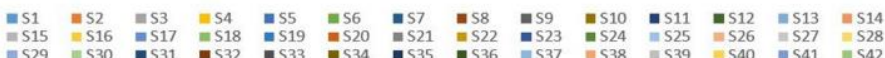

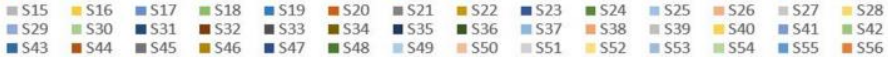

